\title{
Dysbiosis, gut-blood barrier rupture and autoimmune response in rheumatoid arthritis and schizophrenia
}

\author{
Maria Maślińska' ${ }^{\text {ID }}$, Beata Trędzbor ${ }^{\text {ID }}$, Marek Krzystanek² ${ }^{\text {ID }}$ \\ ${ }^{1}$ Early Arthritis Clinic, National Institute of Geriatrics, Rheumatology and Rehabilitation, Warsaw, Poland \\ 2Department and Clinic of Psychiatric Rehabilitation, Department of Psychiatry and Psychotherapy, Faculty of Medical Sciences, \\ Medical University of Silesia in Katowice, Poland
}

\begin{abstract}
The primary cause of chronic autoimmune diseases is elusive both in somatic medicine and psychiatry. Examples of such conditions are rheumatoid arthritis and schizophrenic disorders. Immune disturbances occur in both diseases, but it is difficult to combine them into a meaningful pathogenetic model. The immunological hypothesis of schizophrenia is based on non-specific changes in the cytokine system and exponents of chronic inflammation in some patients. In rheumatoid arthritis the cytokine network is much better known than in schizophrenia, and interleukin-6, tumor necrosis factor or Janus kinases became a target of treatment. Microbiome dysbiosis and disturbances of the blood-gut barrier may be a new hypothesis of the pathogenesis of somatic and psychiatric diseases. The purpose of this narrative review was to show, using the example of two chronic diseases - rheumatoid arthritis and schizophrenic disorders - that disturbances in the blood barrier of the intestine can be a common mechanism of somatic and mental disorders.

The paper presents the current state of knowledge on the hypothetical relationship between microbiome dysbiosis and the pathogenesis of schizophrenia and rheumatoid arthritis. In conclusion, in the light of discoveries regarding the microbiome-gut-brain axis the immunological model of rheumatoid arthritis and schizophrenia formation may gain importance and contribute to the creation of new strategies for causal treatment of these still incurable diseases.
\end{abstract}

Key words: rheumatoid arthritis, schizophrenia, microbiome dysbiosis, autoimmune modulation, gastrointestinal-blood barrier.

\section{Introduction}

Both rheumatology and psychiatry have a large portfolio of incurable diseases. In this context, new hypotheses and models of these diseases are of interest, indicating the possibility of their causal and perhaps also effective treatment. These diseases are certainly rheumatoid arthritis (RA) for rheumatology and schizophrenia for psychiatry.

Rheumatoid arthritis is a good model of an autoimmune disease as its immunological system disturbances are better known than in other rheumatic diseases. The inflammatory process in RA affect mainly the structures of the joints. However, genetic susceptibility (e.g.
HLA-DRB1 and PTPN2) as well as environmental factors such as ultraviolet radiation, hormones, infections, especially induced by oral pathogens, Porphyromonas gingivalis, for instance, which is associated with periodontitis, are the major risk factors in RA development [1, 2]. Among habits, nicotine smoking plays a major role in RA development [1]. The abovelisted factors influence the process of protein carbamylation and citrullination, which causes inflammation and damage of joint structures $[1,2]$.

Some clinical and immunological factors such as female gender, rapid onset of the disease, polyarthritis, as well as laboratory tests - high serum concentration of $C$ reactive protein (CRP), elevated erythrocyte sedimen-

Address for correspondence:

Maria Maślinska, Early Arthritis Clinic, National Institute of Geriatrics, Rheumatology and Rehabilitation, 1 Spartanska St.,

02-637 Warsaw, Poland, e-mail: maslinskam@gmail.com

Submitted: 08.03.2021; Accepted: 17.06.2021 
tation rate (ESR), and the occurrence of both rheumatoid factor (RF) and anti-citrullinated proteins antibodies (ACPA) - were established as indicators of poor prognosis and prompt the clinicians to treat more aggressively, even in the early stage of the disease [3]. In recent years the importance of the gut microbiome, especially dys biosis, has been highlighted as a significant factor in the development and modulation of RA [4].

In contrast to the established pathophysiology and autoimmune response in RA, the discussion about the autoimmune background of schizophrenia has been ongoing for years. The influence of infections and disturbances of the glutamatergic system, which is related to the immune system, is indicated as a potential cause and effector of disorders occurring in this disease [5]. Schizophrenia is a group of diseases with probably different etiologies that produce similar clinical symptoms. In the formation of schizophrenia, the diathesis-stress model has been currently adopted from psychology, in which various environmental factors trigger genetic susceptibility to illness [5-7]. One of the unproven and pending hypotheses for schizophrenic disorders is that it is caused by immune disturbances.

The hypothesis of immune dysfunction in schizophrenia is based, among others, on genetic premises. GWAS associative studies indicate that proteins involved in the inflammatory reaction, encoded in chromosome 6 regions (6p21-6p22) and containing sequences of about four hundred genes of the major histocompatibility complex (MHC), are associated with the risk of schizophrenia and synapse formation [8-10].

Immune dysfunction underlying schizophrenia is hypothesized to be either an element of the complex pathomechanism of schizophrenia or, more likely, a model of one type of schizophrenia with chronic inflammatory markers. Several studies indicate that dysfunction in the immune system affects the most severe forms of schizophrenia associated with poor prognosis [11, 12]. One proposed mechanism to immunize the body with antigens that could then play a role in the pathogenesis of schizophrenia is the break of the gastrointestinal-blood barrier in the stomach and intestines. Immunological disturbances are present in both RA and schizophrenia, but each of these diseases lacks a consistent and complete concept of its underlying causes as well as an understandable hypothetical model of the pathogenesis of the disease, especially schizophrenia.

The authors decided to use the example of these two chronic diseases to present, in the form of a narrative review, the evidence that microbiome dysbiosis and disturbances of the gastrointestinal-blood barrier may be the basis of a new model of pathogenesis of these diseases. The confirmation of this model may be the starting point for the development and research of new, causal strategies for the treatment of chronic diseases in both somatic medicine and psychiatry.

\section{Outline of immunological disturbances in rheumatoid arthritis}

The model of RA pathogenesis is constantly updated with new elements explored thanks to the development of laboratory techniques, imaging tests and clinical observations.

According to current knowledge RA can be differentiated into the pre-RA stage, early RA and fully developed RA. Obviously, expanding knowledge guides clinicians towards early diagnosis; however, pre-RA remains in the realm of scientific inquiry, despite striving for optimal screening and selecting people at risk of the disease. Pre-RA is currently not recognized in everyday practice. Pre-RA is defined as a state with arthralgia and with no clinical features of arthritis, with presence of high concentrations of ACPA antibodies and RF or the presence of a high concentration of ACPA antibodies alone [13]. It was observed that $40 \%$ of patients from this group develop RA within 18 months [13].

The microbiome influence on inflammation in RA is an important discovery and a hypothesis proven in the latest research. The pathway between the oral cavity, intestines and joints seems long, but recent studies confirm the role of the microbiome as well as the imbalance between symbiotes and commensals, and the presence of pathogens in the pathogenesis and the course of RA [14].

So far, there is no precise definition of what the balance in the proportion of bacteria that the microbiome should consist of should be and what criteria to adopt for microbiome disorders. However, the research conducted so far suggests that imbalances in the physiological microbiome, i.e. microbiome dysbiosis, may lead to disturbances with interstitial permeability, molecular mimicry, and post-translational modifications, which lead to stimulation of the inflammatory response with production of proinflammatory cytokines (IL-6, IL-1B, TNF- $\alpha, I L-17 A$ ) and the presence of ACPA and RF in joint space and patients' serum.

\section{Immunological disturbances in schizophrenia}

The immunological hypothesis of schizophrenia is based on observations indicating the presence of markers of chronic inflammation in some patients suffering from schizophrenia. This hypothesis has not been confirmed, and the mechanisms that can connect the immune system to processes in the central nervous system (CNS) are still unknown. However, it is known that some 
cytokines that may be elevated in schizophrenia, such as TNF- $\alpha$, IL- $1 \beta$ and IL-6, may also play an important role in the central nervous system by modulating neuronal function [15].

Immune disturbances in schizophrenia are complex and research results are often inconsistent. A recent meta-analysis by Çakici et al. [16] indicated that in schizophrenia, compared to healthy people, the levels of interleukin 6, 8 and tumor necrosis factor $\alpha$ are increased, there is a trend of increased levels in the levels of acute phase protein, interferon $\gamma$ and interleukin 4, and there are no changes in the level of interleukin $\beta$, 2 , 10, 12, monocyte chemoattractant protein-1 and transforming growth factor $\beta$.

An important argument for the immunological hypothesis of schizophrenia is the role of MHC proteins in the development of the CNS. The primary function of MHC proteins, encoded by the human leukocyte antigen ( $H L A)$, is the presentation of antigens in the circulatory system to preserve the immunological integrity of the body. It turns out that MHC proteins also have an important function in the central nervous system (CNS). Animal studies have shown that MHC antigens are involved in processes related to the development of the nervous system, such as neurogenesis, neuronal migration and pruning [17]. This mainly occurs in the early stage of animal CNS development.

The link between HLA genes and neurotropic virus infections also indirectly supports the malfunction of the immune system in schizophrenia. Infections with these viruses are associated with an increased risk of schizophrenia, and patients with schizophrenia have been shown to have a chronic increase in the level of antibodies specific for these infections [18]. Polymorphisms in HLA genes have been shown to be associated with infection with neurotropic viruses such as cytomegalovirus or type I of herpes simplex virus.

HLA genes have also been successfully linked to the pathological function of the gut-blood barrier. Namely, the relationship between HLA genes and gastrointestinal diseases associated with inflammatory processes in the intestinal mucosa and damage to the gut-blood barrier has been proven. The foremost representatives of these diseases are ulcerative colitis, celiac disease and Crohn's disease $[19,20]$.

This means that antigens and pathogens that penetrate the damaged intestinal-blood barrier can cause immunization of the body. This immunization in people with a genetic susceptibility may lead to incorrect recognition of MHC antigens and cause an abnormal autoimmune response that can even reach the nervous system [21].

Interestingly, the expression of MCG genes in the CNS on neurons and astrocytes is highest in the early stages of life, which would mean that it is the period of fetal life and youth that may be at greatest risk for the manifestation of immune system abnormalities in schizophrenia and autoimmunity. This is consistent with the neurodevelopmental model of schizophrenia, which posits that the process of schizophrenia is responsible for the interaction of genetic susceptibility with the action of various environmental factors at the stage of neurogenesis in fetal life and pruning in childhood, adolescence and early youth.

Another important player in the immune theory of schizophrenia is the complement system, which supports and directs the immune system to defend the genetic integrity of the body. In schizophrenia, it has recently been shown that the number of copies of the gene encoding the C4 component of the complement system is associated with a higher risk of schizophrenia [22]. Moreover, it has been shown that a larger C4 copy number is directly related to the weakening of synaptogenesis and increased CNS pruning [23].

This pilot study was conducted on young schizophrenic patients in whom pruning is still an active phase of nervous system maturation. This means that the increased immune response during childhood, adolescence and early adulthood can interfere with the maturation of the nervous system, which may be the cause of diseases such as schizophrenia or other nervous system diseases [24].

Recent research on the role of the gut-brain axis in the development of schizophrenia shows the immunological hypothesis of schizophrenia in a new light, creating a new, coherent and practical model of the pathogenesis of this disease.

\section{Gut model of rheumatoid arthritis}

As already mentioned in the section on the pathogenesis of RA, the role of the microbiome is now recognized as particularly important in the pathogenesis of this autoimmune disease. Initially, the focus was on the influence of Porphyromonas gingivalis infection in the oral cavity on the process of protein citrullination and the formation of ACPA antibodies, but now the entire intestinal area and changes in its microbiome are being analyzed. Antibodies to $P$. gingivalis have been shown to play a role in the development of arthritis together with other risk factors for RA development such as smoking and genetic predisposition (HLA-DRB1 shared epitope alleles) [25].

In patients with RA, the growth of Prevotella and $P$. copri species was observed significantly more often in preclinical and early RA compared to healthy controls. However, further research is needed to investigate their role in the transition from the preclinical to the clinical stage of RA. 
A significant expansion of Bacilli class and Lactobacillales was demonstrated in RA as well as the expansion of Lactobacillus salivarius in oral and intestinal samples, and the abundance of bacteria was related to disease severity in RA patients. The inverse observations were also reported in collagen-induced arthritis after oral administration of $L$. salivarius, in which a decrease in bone erosions and synovial infiltration with activation of Treg cells and increased production of IL-10 levels was observed [26].

When analyzing the role of the intestinal microbiome in the development and course of RA, attention should be paid to the fact that microbial diversity is diminishing and that it will affect a number of regulatory mechanisms and the immune response.

Post-translational modifications of host proteins are known, especially in oral bacteria such as Aggregatibacter actinomycetemcomitans (A.a.) and Porphyromonas gingivalis augment, through the action of leukotoxin-A (Ltx-A) in the case of A.a. and Porphyromonas-PAD (PPAD) enzyme activity ( $P$. gingivalis). Prevotella spp. has $N$-acetylglucosamine-6-sulfatase (GNS), which is structurally similar to an RA-citrullinated autoantigen, which has ability to activate the $T$ and $B$ cell response. This is an example of a molecular mimicry mechanism related to the gut flora and RA [26].

The third important way the microbiome influences the immune process is through the development of an inflammatory response. In experimental arthritis, the influence of P. copri and Lactobacillus species on CD4+ T cells has been demonstrated, particularly by increasing the numbers of IL-17+ Th17 cells and activating Th1 cell responses.

The importance of intestinal barrier permeability in the situation of dysbiosis, as demonstrated in animal models, cannot be ignored either. Collinsella aerofaciens was shown to promote intestinal permeability by increas- ing inflammatory mediators and chemokines (IL-17A, CXCL1, CXCL5) and reducing the expression of tight junction proteins (ZO-1 and occludin), while colonization with Prevotella copri induced colitis in mice by increasing CD4+ (IFN- $\gamma+)$ T cells $[26,27]$. The consequence of the loss of protection of the intestinal barrier is the translocation of antigens for circulation and activation of the parenteral inflammatory process, including in the joints.

The gut microbiome is not stable and intrinsic and extrinsic stimuli can change the host reaction. Modulation by use of antibiotics and prebiotics or fecal microbiota transplant (FMT) may be involved in modulation of activation of the host response and inflammatory process. The microbiome composition can also be influenced through dietary intake.

Figure 1 presents an outline of mechanisms by which altered gut microbiota affects the development of RA.

\section{Gut model of schizophrenia}

There is a physiological and pathological gut-brain axis in the human body. The physiological axis is associated with the function of the visceral brain, which communicates bidirectionally with the CNS through hormones and the vagus nerve. In turn, the pathological gut-brain axis refers to inflammatory changes caused by damage to the gut-blood barrier by a microbiome or toxins such as gluten followed by immunization of the body, which can cause similar autoimmune and neuroinflammatory processes in the CNS.

The gastrointestinal mucosa is a barrier between the body and the external environment. In the intestinal mucosa, processes occur that determine the integrity of the body's internal environment and the impermeability of the blood-intestine barrier, while at the interface
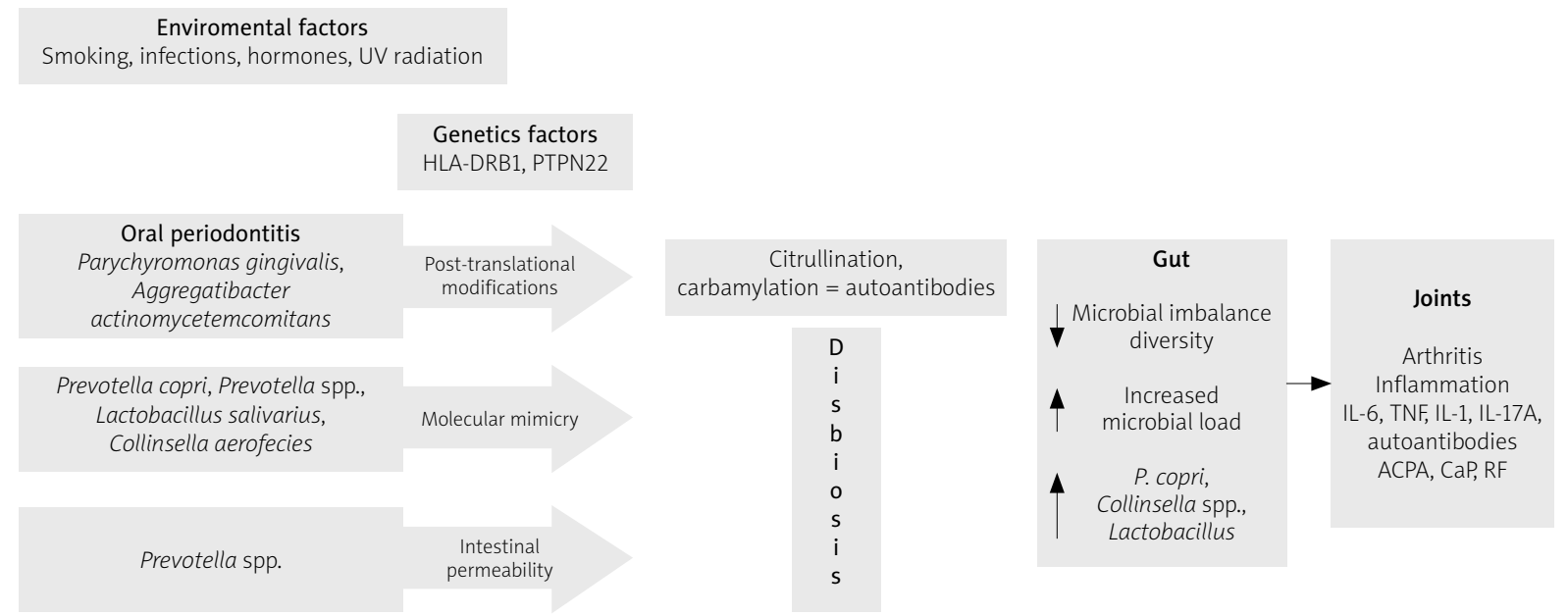

Fig. 1. Outline of mechanisms by which altered gut microbiota affects the development of rheumatoid arthritis $[4,26]$. 
between the two environments a drama of defense against pathogens and toxins, the formation of immune immunity and the inflammatory process take place.

Dysbiosis, or disturbances in the proportion of the physiological composition of the microbiome, plays an important role in the mucosa alterations. This problem is still being investigated, but it has already been observed that compared to healthy controls in the stool of patients with schizophrenia, there are higher numbers of Proteobacteria, Succinivibrio, Megasphaera, Collinsella, Clostridium, Klebsiella and Methanobrevibacter, and lower numbers of Blautia, Coprococcus and Roseburia [28].

The microbiota-gut-brain (MGB) schizophrenia model assumes that the gastric and intestinal mucosa, together with physiological flora, form a barrier separating the body from the external environment and allow the body to maintain immune homeostasis in the meaning of defense processes against foreign antigens. People who are carriers of the alleles of predisposition to immune disturbances, when exposed to environmental factors such as diet, bacterial or viral infection, drugs or toxins, may develop microbial imbalances in the digestive system and intestinal-blood barrier damage.

Such exposure may occur as early as in the prenatal period, during delivery or after delivery through food, drugs or toxins. As a result, an inflammatory process may occur in the intestinal mucosa, during which bacterial antigens may pass through the intestinal barrier into the blood, inducing an inflammatory and immune response of the body [11]. The same antigens can pass through the blood-brain barrier, causing an inflammatory process in the central nervous system that interferes with brain development and function.

In addition, there is a risk that the body may develop an autoimmune response against its own antigens, i.e. an autoimmune response that targets MHC antigens, also represented on neurons, astrocytes and microglia in the CNS or against NMDA receptor subunit pro-

Neuron

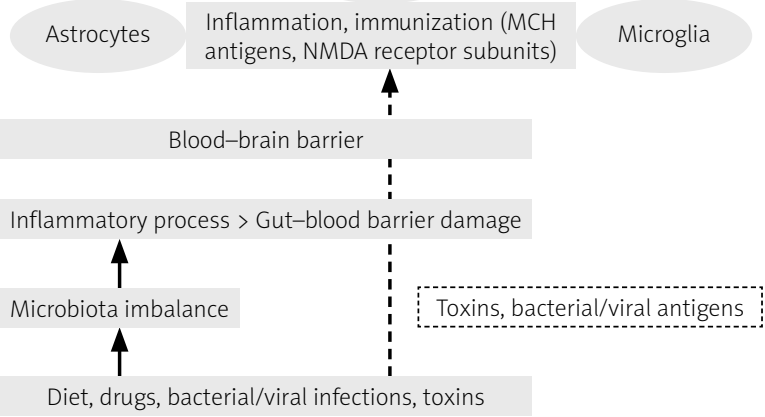

Fig 2. Outline of the microbiome disturbance in schizophrenia. teins, key for the pathogenesis of schizophrenia [29, 30]. The deciding factor is whether the body is able to correctly recognize foreign antigens and fight them. One of the mechanisms of schizophrenia formation may be incorrect pathogen identification and autoimmunity, which can lead to inflammation within the CNS [21].

It is assumed that environmental factors disturbing the balance of the microbiome may act both in the fetal period and in the early periods of life, leading to the formation of abnormal bacterial flora, and ultimately to a chronic inflammatory process. Therefore, the hypothesis of a relationship between dysbiosis and schizophrenia could be part of the neurodevelopmental hypothesis of schizophrenia. It has been proven that fetal exposure to the mother's immune process is a risk factor for schizophrenia, child development disorders as well as biochemical disturbances in the brain [31]. Vertical transfer of the maternal inflammatory microbiome to offspring can cause inflammation in the intestine of the fetus, and lead to an inflammatory reaction and immunity of the body at a very early stage of life.

The hypothesis of a relationship between dysbiosis and inflammation in the gut and brain is mainly based on evidence obtained from experimental studies on rodents. Microbiome composition disturbances have been found to have a direct impact on CNS processes associated with neurogenesis, synaptic plasticity, or pruning [32, 33]. Furthermore, the transplantation of the microbiome of people with schizophrenia into mice causes significant biochemical disturbances in the field of GABAergic and glutaminergic system activity - systems that play an important role in the pathophysiology of schizophrenia [34].

In humans, the test results are not yet consistent, and the results obtained in case-control studies indicating similar inflammatory processes in the CNS and in the intestinal mucosa are ambiguous and may be an artefact or accidental coincidence. Disorders in the blood cytokine system of people with schizophrenia may be a derivative of inflammatory processes in the CNS, associated not only with the MGB axis, but also with excitotoxicity as part of the pathogenetic process of schizophrenia, as well as the result of the action of drugs or various environmental influences such as stress, medicine or chemical pollution. This is a problem that has not been clarified by science and requires further research. Figure 2 presents a diagram of the outline of the microbiome disturbance in schizophrenia.

\section{The association between schizophrenia and rheumatoid arthritis}

In some studies, an inverse association between RA and schizophrenia has been described [35], but the differences between RA and schizophrenic patients in 
terms of age (usually older than schizophrenic patients) as well as the differences between seropositive and seronegative types of RA and coexistence of schizophrenia were raised. The differences were also explained by the different genetic background of the two diseases [36].

Depression and anxiety are significantly associated with RA. There is scientific evidence of genetic links (genome-wide association studies - GWAS) between chronic/persistent inflammation symptoms and neurodegeneration. It is therefore considered that RA may increase the risk of neurodegenerative diseases in the future life of the patients [37].

\section{Gut-brain axis in rheumatoid arthritis}

The gut-brain axis (GBA) can be understood as a communication network or cross-talk between the CNS and enteric nervous system (ENS) [38].

In RA peripheral inflammatory signals (e.g. proinflammatory cytokines, kinase pathway) must enter the CNS by the autonomic nervous system. One of the most important efferent routes is the vagus nerve, which controls production of TNF and other pro-inflammatory cytokines. This is possible due to the splenic nerve, which activates $\beta$-adrenergic receptors on splenic CD4+ T cells (choline acetyltransferase positive, ChAT+) with the ability through cholinergic signaling to suppress other immune cells to produce cytokine [38]. The inflammatory signals from the periphery activate CNS-resident cells, particularly microglia and astrocytes, which is suggested to be the cause of maintaining a neuroinflammatory state in RA. These phenomena are more widely studied in animal models, but are undoubtedly not yet fully understood [38, 39].

The activation of GBA in RA has been taken into account as a risk factor for the neuroinflammatory process.

Neuropsychiatric comorbidity in RA substantially contributes to disease burden and worsens the therapeutic response and outcome; on the other hand, the anti-inflammatory treatment of RA relieves neuropsychiatric symptoms such as depression and anxiety [38]. This may prove the importance of the gut-brain axis in RA and provide the basis for a discussion on further therapeutic targets in this disease.

\section{Diet, probiotics and treatment Rheumatoid arthritis}

The influence of diet on the development of RA is controversial. It seems that the role of omega- 3 acids has been proven [40]. The Mediterranean diet is a widely recognized beneficial pattern of nutrition in inflammatory diseases, including RA [41, 42]. Apart from omega-3 acids, the immunomodulatory effect of fiber has been demonstrated [38].
The Western model of a diet with a high intake of saturated fat is expected to promote intestinal permeability and thus enable the movement of bacterial lipopolysaccharides (LPS) into the systemic circulation and stimulate the inflammatory process. It is now known that obesity is one of the factors in the development of RA because adipose tissue releases, among other things, adipokines such as leptin and inflammatory cytokines such as TNF- $\alpha$, IL-1 $\beta$ and IL- 6 , which may contribute to the development and severity of an inflammatory response [43].

The diet may contain compounds such as capsaicin from chili or curcumin from Curcuma longa, both compounds with immunomodulatory and antioxidant as well as anti-inflammatory properties by activating TRPV1 receptors (capsaicin) on T lymphocytes [44] or by inhibiting nuclear transcription factor $\kappa \mathrm{B}(\mathrm{NF}-\kappa \mathrm{B})$, cyclooxygenase 2 (COX-2) and inducible nitric oxide synthase (iNOS) (curcumin) [45].

There is a lot of research on the role of vitamin $D$ as a regulator of target genes' expression, factors stimulating and inhibiting inflammatory and autoimmune processes by influencing the proliferation of B cells and autoantibody production, inhibiting the release of Th1 and Th17 cytokines [46, 47].

Fiber, on the other hand, promotes the production of intestinal short-chain fatty acids by the intestinal microflora, which has anti-inflammatory and beneficial metabolic effects. Recognizing the problem of intestinal dysbiosis as important in the pathogenesis of $\mathrm{RA}$, it is possible to design an appropriate diet that can restore the proper balance of the intestinal microflora in this group of RA patients, e.g. a study NCT02941055, NCT04262505 [48, 49].

An additional benefit of inhibiting the inflammatory process in this way and obtaining a favorable metabolic profile would be the reduction of cardiovascular risk in patients with RA.

Probiotics in RA treatment are also discussed. The hypothesis of benefits from stabilization of the hostmicrobe relationship, which can potentially reduce the inflammatory process, is increasingly discussed and analyzed as a possibility for additive therapy. There are randomized controlled trials which show that probiotics may reduce the concentration of proinflammatory cytokines, but the clinical effect of probiotics on RA activity remains not fully confirmed, and there is currently no consensus on the effectiveness of probiotics in treatment of arthritis [50].

\section{Schizophrenia}

A proposal for a new model of schizophrenia development in the pathological microbiota-gut-brain axis may be the starting point for new treatment strategies. 
The intervention may consist of treating dysbiosis itself as a source of inflammation in the intestines, treating inflammation as a symptom, or autoimmune treatment. Previous attempts to administer probiotics to schizophrenia patients have not resulted in any significant changes in their symptoms [51,52].

Vitamin D deficiency also affects the severity of the disease; therefore the assessment and maintenance of its normal level should also be taken into account when determining the patient's diet [53].

It is possible, however, that such intervention takes place too late. In fact, it should take place at an early stage of life in children who have been identified as having exponents of the inflammatory process or genetic variants associated with susceptibility to schizophrenia. However, the introduction of this type of early-intestinal intervention in schizophrenia requires further research. By understanding the role of the microbiome, new perspectives in the treatment of schizophrenia are emerging.

\section{Conclusions}

Rheumatoid arthritis and schizophrenia are auto immune diseases in which the role of the microbiome is increasingly better understood. The gut-brain axis, which is better described in schizophrenia, may also be important in RA.

Due to the existence of specific biomarkers, RA is now better known in terms of autoimmune pathogenesis and even has a mathematical algorithm for diagnosis (classification criteria).

The microbiome, through its influence on post-translational processes, has an impact on the formation of ACPA autoantibodies in RA. In schizophrenia, dysbiosis may influence GABAergic and glutaminergic system activity, which may underlie the pathogenesis of this disease, cause its deterioration and determine the severity of the disease.

The authors declare no conflict of interest.

\section{References}

1. Deane KD, Demoruelle MK, Kelmenson LB, et al. Genetic and environmental risk factors for rheumatoid arthritis. Best Pract Res Clin Rheumatol 2017; 31: 3-18, DOI: 10.1016/j.berh. 2017.08.003.

2. Lundberg K, Wegner N, Yucel-Lindberg T, Venables PJ. Periodontitis in RA-the citrullinated enolase connection. Nat Rev Rheumatol 2010; 6: 727-730, DOI: 10.1038/nrrheum.2010.139.

3. Albrecht K, Zink A. Poor prognostic factors guiding treatment decisions in rheumatoid arthritis patients: a review of data from randomized clinical trials and cohort studies. Arthritis Res Ther 2017; 19: 68, DOI: 10.1186/s13075-017-1266-4.

4. Rooney CM, Mankia K, Emery P. The role of the microbiome in driving RA-related autoimmunity. Front Cell Dev Biol 2020; 8: 538130, DOI: 10.3389/fcell.2020.538130.
5. Müller N, Schwarz JM. The role of immune system in schizophrenia. Curr Immunol Rev 2010; 6: 213-220, DOI: 10.2174/ 157339510791823673.

6. Demjaha A, MacCabe JH, Murray RM. How genes and environmental factors determine the different neurodevelopmental trajectories of schizophrenia and bipolar disorder. Schizophr Bull 2012; 38: 209-214, DOI: 10.1093/schbul/sbr100.

7. Van Os J, Rutten BP, Myin-Germeys I, et al. European Network of National Networks studying Gene-Environment Interactions in Schizophrenia. Identifying gene-environment interactions in schizophrenia: contemporary challenges for integrated, large-scale investigations. Schizophr Bull 2014; 40 : 729-736, DOI: 10.1093/schbul/sbu069.

8. International Schizophrenia Consortium; Purcell SM, Wray NR, Stone JL, et al. Common polygenic variation contributes to risk of schizophrenia and bipolar disorder. Nature 2009; 460: 748-752, DOI: 10.1038/nature08185.

9. Jones AL, Mowry BJ, Pender MP, Greer JM. Immune dysregulation and self-reactivity in schizophrenia: do some cases of schizophrenia have an autoimmune basis? Immunol Cell Biol 2005; 83: 9-17, DOI: 10.1111/j.1440-1711.2005.01305.x.

10. Boulanger LM. Immune proteins in brain development and synaptic plasticity. Neuron 2009; 64: 93-109, DOI: 10.1016/ j.neuron.2009.09.001.

11. Abegunde AT, Muhammad BH, Bhatti O, Ali T. Environmental risk factors for inflammatory bowel diseases: evidence based literature review. World J Gastroenterol 2016; 22: 6296-6317, DOI: 10.3748/wjg.v22.i27.6296.

12. Fond G, Godin O, Boyer L, et al. Chronic low-grade peripheral inflammation is associated with ultra resistant schizophrenia. Results from the FACE-SZ cohort. Eur Arch Psychiatry Clin Neurosci 2018; 269: 985-992, DOI: 10.1007/s00406-018-0908-0.

13. Paul BJ, Kandy HI, Krishnan V. Pre-rheumatoid arthritis and its prevention. Eur J Rheumatol 2017; 4: 161-165, DOI: 10.5152/ eurjrheum.2017.16006.

14. Maeda Y, Takeda K. Role of gut microbiota in rheumatoid arthritis. J Clin Med 2017; 6: 60, DOI: 10.3390/jcm6060060.

15. Ramírez-Jirano LJ, Velasco-Ramírez SF, Pérez-Carranza GA, et al. Cytokines and nervous system: relationship with schizophrenia. Rev Med Inst Mex Seguro Soc 2019; 57: 107-112.

16. Çakici N, Sutterland AL, Penninx BWJH, et al. Altered peripheral blood compounds in drug-naïve first-episode patients with either schizophrenia or major depressive disorder: a metaanalysis. Brain Behav Immun 2020; 88: 547-558, DOI: 10.1016/j.bbi.2020.04.039.

17. McAllister AK. Major histocompatibility complex in brain development and schizophrenia. Biol Psychiatry 2014; 75: 262-268, DOI: 10.1016/j.biopsych.2013.10.003.

18. Hornig M. The role of microbes and autoimmunity in the pathogenesis of neuropsychiatric illness. Curr Opin Rheumatol 2013; 25: 488-795, DOI: 10.1097/BOR.0b013e32836208de.

19. Cukrowska B, Sowinska A, Bierla JB, et al. Intestinal epithelium, intraepithelial lymphocytes and the gut microbiota - key players in the pathogenesis of celiac disease. World I Gastroenterol 2017; 23: 7505-7518, DOI: 10.3748/wjg.v23.i42.7505.

20. Gomes RG, Brito CAA, Martinelli VF, et al. HLA-G is expressed in intestinal samples of ulcerative colitis and Crohn's disease patients and HLA-G5 expression is differentially correlated with TNF and IL-10 cytokine expression. Hum Immunol 2018; 79: 477-484, DOI: 10.1016/j.humimm.2018.03.006. 
21. Wekerle $\mathrm{H}$. Brain autoimmunity and intestinal microbiota: 100 trillion game changers. Trends Immunol 2017; 38: 483-497, DOI: 10.1016/j.it.2017.03.008.

22. Sekar A, Bialas AR, de Rivera $H$, et al. Schizophrenia risk from complex variation of complement component 4. Nature 2016; 530: 177-183, DOI:10.1038/nature16549.

23. Prasad KM, Chowdari KV, D'Aiuto LA, et al. Neuropil contraction in relation to complement c4 gene copy numbers in independent cohorts of adolescent-onset and young adult-onset schizophrenia patients - a pilot study. Transl Psychiatry 2018 8: 134, DOI: 10.1038/s41398-018-0181-z.

24. Bernstein CN, Hitchon CA, Walld R, et al. Increased burden of psychiatric disorders in inflammatory bowel disease. Inflamm Bowel Dis 2018; 25: 360-368, DOI: 10.1093/ibd/izy235.

25. Kharlamova N, Jiang $X$, Sherina N, et al. Antibodies to Porphyromonas gingivalis indicate interaction between oral infection, smoking, and risk genes in rheumatoid arthritis etiology. Arthritis Rheumatol 2016; 68: 604-613, DOI: 10.1002/art.39491.

26. Maeda Y, Takeda K. Host-microbiota interactions in rheumatoid arthritis. Exp Mol Med 2019; 51: 1-6, DOI: 10.1038/s12276019-0283-6.

27. Zhang X, Zhang D, Jia H, et al. The oral and gut microbiomes are perturbed in rheumatoid arthritis and partly normalized after treatment. Nat Med 2015; 21: 895-905, DOI: 10.1038/nm.3914.

28. Shen Y, XU J, Li Z, et al. Analysis of gut microbiota diversity and auxiliary diagnosis as a biomarker in patients with schizophrenia: a cross-sectional study. Schizophr Res 2018; 197: 470-477, DOI: 10.1016/j.schres.2018.01.002

29. Kannan G, Gressitt KL, Yang S, et al. Pathogen-mediated NMDA receptor autoimmunity and cellular barrier dysfunction in schizophrenia. Transl Psychiatry 2017; 7: e1186, DOI: 10.1038/ tp.2017.162.

30. Balu DT. The NMDA receptor and schizophrenia: from pathophysiology to treatment. Adv Pharmacol 2016; 76: 351-382, DOI: 10.1016/bs.apha.2016.01.006

31. Severance EG, Yolken RH. Deciphering microbiome and neuroactive immune gene interactions in schizophrenia. Neurobiol Dis 2020; 135: 104331, DOI: 10.1016/j.nbd.2018.11.016.

32. Erny D, Hrabé de Angelis AL, Jaitin D, et al. Host microbiota constantly control maturation and function of microglia in the CNS. Nat Neurosci 2015; 18: 965-977, DOI: 10.1038/nn.4030.

33. Luczynski P, Whelan SO, O’Sullivan C, et al. Adult microbiotadeficient mice have distinct dendritic morphological changes: differential effects in the amygdala and hippocampus. Eur J Neurosci 2016; 44: 2654-2666, DOI: 10.1111/ejn.13291.

34. Sampson TR, Mazmanian SK. Control of brain development, function, and behavior by the microbiome. Cell Host Microbe 2015; 17: 565-576, DOI: 10.1016/j.chom.2015.04.011.

33. Zheng $P$, Zeng $B$, Liu $M$, et al. The gut microbiome from patients with schizophrenia modulates the glutamate-glutamine-GABA cycle and schizophrenia-relevant behaviors in mice. Sci Adv 2019; 5: eaau8317, DOI: 10.1126/sciadv.aau8317.

35. Chen SF, Wang LY, Chiang J, et al. Assessing whether the association between rheumatoid arthritis and schizophrenia is bidirectional: a nationwide population-based cohort study. Sc Rep 2019; 9: 4493, DOI: 10.1038/s41598-018-38149-3.

36. Sellgren C, Frisell T, Lichtenstein P, et al. The association between schizophrenia and rheumatoid arthritis: a nationwide population-based Swedish study on intraindividual and familial risks. Schizophr Bull 2014; 40: 1552-1559, DOI: 10.1093/ schbul/sbu054.
37. Broce I, Karch CM, Wen N, et al. Immune-related genetic enrichment in frontotemporal dementia: An analysis of genome-wide association studies. PLoS Med 2018; 15: e1002487, DOI: 10.1371/ journal.pmed.1002487.

38. Süß P, Rothe T, Hoffmann A, et al. The joint-brain axis: insights from rheumatoid arthritis on the crosstalk between chronic peripheral inflammation and the brain. Front Immunol 2020; 11: 612104, DOI: 10.3389/fimmu.2020.612104.

39. El Aidy S, Dinan TG, Cryan JF. Immune modulation of the braingut-microbe axis. Front Microbiol 2014; 5: 146, DOI: 10.3389/ fmicb.2014.00146.

40. Chavan SS, Pavlov VA, Tracey KJ. Mechanisms and therapeutic relevance of neuro-immune communication. Immunity 2017; 46: 927-942, DOI: 10.1016/j.immuni.2017.06.008.

41. Kwiatkowska B, Maślińska M. The place of omega-3 and omega- 6 acids in supplementary treatment of inflammatory joint diseases. Reumatologia 2020; 58: 34-41, DOI: 10.5114/ reum.2020.93511

42. Picchianti Diamanti A, Panebianco C, Salerno G, et al. Impact of Mediterranean diet on disease activity and gut microbiota composition of rheumatoid arthritis patients. Microorganisms 2020; 8: 1989, DOI: 10.3390/microorganisms8121989.

43. Stavropoulos-Kalinoglou A, Metsios GS, Koutedakis Y, Kitas GD. Obesity in rheumatoid arthritis. Rheumatology (Oxford) 2011; 50: 450-462, DOI: 10.1093/rheumatology/keq266.

44. Yang F, Zheng J. Understand spiciness: mechanism of TRPV1 channel activation by capsaicin. Protein Cell 2017; 8: 169-177, DOI: 10.1007/s13238-016-0353-7.

45. Basnet P, Skalko-Basnet N. Curcumin: an anti-inflammatory molecule from a curry spice on the path to cancer treatment. Molecules 2011; 16: 4567-4598, DOI: 10.3390/molecules16064567.

46. Yang CY, Leung PS, Adamopoulos IE, Gershwin ME. The implication of vitamin $D$ and autoimmunity: a comprehensive review. Clin Rev Allergy Immunol 2013; 45: 217-226, DOI: 10.1007/ s12016-013-8361-3.

47. Kostoglou-Athanassiou I, Athanassiou P, Lyraki A, et al. Vitamin D and rheumatoid arthritis. Ther Adv Endocrinol Metab 2012; 3: 181-187, DOI: 10.1177/2042018812471070.

48. Vadell AKE, Bärebring L, Hulander E, et al. Anti-inflammatory Diet In Rheumatoid Arthritis (ADIRA) - a randomized, controlled crossover trial indicating effects on disease activity. Am J Clin Nutr 2020; 111: 1203-1213, DOI: 10.1093/ajcn/nqaa019.

49. Häger J, Bang $H$, Hagen $M$, et al. The role of dietary fiber in rheumatoid arthritis patients: a feasibility study. Nutrients 2019; 11: 2392, DOI: 10.3390/nu11102392.

50. Milajerdi A, Mousavi SM, Sadeghi A, et al. The effect of probiotics on inflammatory biomarkers: a meta-analysis of randomized clinical trials. Eur J Nutr 2020; 59: 633-649, DOI: 10.1007/s00394-019-01931-8

51. Pełka-Wysiecka J, Kaczmarczyk M, Bąba-Kubiś A, et al. Analysis of gut microbiota and their metabolic potential in patients with schizophrenia treated with olanzapine: results from a six-week observational prospective cohort study. J Clin Med 2019; 8: pii: E1605, DOI: 10.3390/jcm8101605.

52. Ng QX, Soh AYS, Venkatanarayanan N, et al. A systematic review of the effect of probiotic supplementation on schizophrenia symptoms. Neuropsychobiology 2019; 78: 1-6, DOI: 10.1159/000498862.

53. Doğan Bulut S, Bulut S, Görkem Atalan D, et al. The relationship between symptom severity and low vitamin $D$ levels in patients with schizophrenia. PLoS One 2016; 11: e0165284, DOI: 10.1371/journal.pone.0165284 